\title{
CONVERGENCE RATE FOR A LARGE DEVIATION PROBABILITY
}

\author{
DAVID G. KOSTKA
}

\begin{abstract}
It is known that a condition more stringent than a finite variance is needed to show, by a direct application of large deviation estimates, the convergence of the series used in classical proofs of the law of the iterated logarithm. However, the series still converges if the variables have only a finite variance.
\end{abstract}

1. Introduction. Let $\left\{X_{k}\right\}_{k \geqq 1}$ be a sequence of independent, identically distributed random variables with mean zero and variance one; let $S_{n}=X_{1}+\cdots+X_{n}$. It is well known that the law of the iterated logarithm (L.I.L.) for $S_{n}$ holds if and only if the random variables $X_{k}$ have finite variance. Hartman and Wintner [6] showed the sufficiency of a finite variance, and Strassen [11] showed the necessity.

In classical proofs of the L.I.L. a key estimate (see [8, pp. 41-49]) is

$$
P\left(S_{n} / n^{1 / 2} \geqq a_{n}\right)=\exp \left[-\left(a_{n}^{2} / 2\right)(1+o(1))\right]
$$

as $n \uparrow \infty$ where $a_{n}=(1 \pm \varepsilon)(2 \lg \lg n)^{1 / 2}, \varepsilon>0$. Petrov [9] showed that such a large deviation estimate is readily deduced from Berry-Esseen type theorems when the random variables $X_{k}$ satisfy a condition more stringent than a finite variance (also see [7]).

One of the roles of estimate (1.1) (see Gikhman and Skorohod [5, pp. 289-296] and in particular their Theorem 1 on p. 290 for the symmetric case) is to arrive at the upper class result of the L.I.L. by showing that

$$
\sum_{n=1}^{\infty} \frac{1}{n} P\left(S_{n} / n^{1 / 2} \geqq(1+\varepsilon)(2 \lg \lg n)^{1 / 2}\right)<\infty
$$

for all $\varepsilon>0$. Kostka [7] showed that estimate (1.1) implies the random variables $X_{k}$ satisfy a condition more stringent than a finite variance. Thus assuming only a finite variance it is impossible to get the convergence of series (1.2) by the classical manner of bounding each term with estimate (1.1).

Received by the editors March 26, 1973 and, in revised form, July 16, 1973.

AMS (MOS) subject classifications (1970). Primary 60F10; Secondary 60F05, 60G50.

Key words and phrases. Large deviations, law of the iterated logarithm, Skorohod representation, Gaussian tail estimates, law of large numbers.

(c) American Mathematical Society 1974 
The purpose of this note is to show that series (1.2) still converges when the random variables $X_{k}$ have only a finite variance. The method relies on using the Skorohod embedding to represent the random sums $S_{n}$ by Brownian motion evaluated at random times; and then using a law of large number convergence rate on the random times. Davis [3] considered similar series convergence problems. However, his method will not yield the convergence of series (1.2).

2. Convergence of series (1.2). Recent proofs (see [2, pp. 291-292]) of the law of the iterated logarithm rely on the following representation theorem due to Skorohod (see [2, pp. 276-278]).

(2.1) Skorohod embedding. Let $\left\{X_{n}\right\}_{n \geqq 1}$ be independent random variables with the same distribution; make the normalizations $E\left(X_{n}\right)=0$, $E\left(X_{n}^{2}\right)=1$; and let $S_{n}=X_{1}+\cdots+X_{n}$. Then there exists a probability space $(\Omega, \mathscr{B}, P)$ with a Brownian motion $\xi(t)$ (normalized so that $E[\xi(t)]=0 E\left[\xi^{2}(t)\right]=t$ ) and a sequence of nonnegative, independent, identically distributed random variables $\left\{T_{i}\right\}_{i \geqq 1}$ defined on it such that the following conditions hold:

(2.2)(i) $\left\{\xi\left(\sum_{i=1}^{n} T_{i}\right)\right\}_{n \geqq 1}$ has the same distribution as $\left\{S_{n}\right\}_{n \geqq 1}$.

(2.3)(ii) $E\left(T_{n}\right)=E\left(X_{n}^{2}\right)=1$.

To show the convergence of series (1.2), we also need the following theorem and lemma.

(2.4) TheOREm. Assume $E\left(\left|X_{k}\right|\right)<\infty, E\left(X_{k}\right)=\mu$, then

$$
\sum_{n=1}^{\infty} \frac{1}{n} P\left(\left|S_{n}-n \mu\right|>\varepsilon n\right)<\infty \text { for all } \varepsilon>0 .
$$

Proof. See [10] or [1].

(2.5) Lemma. If $\xi$ is standard Brownian motion and $T$ and $b$ are positive values then

$$
P\left(\sup _{0 \leqq t \leqq T} \xi(t) \geqq b\right)=2 P(\xi(T) \geqq b) .
$$

Proof. See [4, pp. 171-172].

We can now prove the convergence of series (1.2).

(2.6) Theorem. If $E\left(X_{k}\right)=0$ and $E\left(X_{k}^{2}\right)=1$ then

$$
\sum_{n=1}^{\infty} n^{-1} P\left(S_{n} / n^{1 / 2} \geqq a_{n}\right)<\infty
$$

where $a_{n}=(1+\varepsilon)(2 \lg \lg n)^{1 / 2}, \varepsilon>0$. 
Proof. If $\xi(t)$ is standard Brownian, then by the above Skorohod embedding there are random times $\left\{T_{i}\right\}_{i \geqq 1}$ such that $\left\{\xi\left(\sum_{i=1}^{n} T_{i}\right)\right\}_{n \geqq 1}$ has the same distribution as $\left\{S_{n}\right\}_{n \geqq 1}$. Thus it is sufficient to show that

$$
\sum_{n=1}^{\infty} n^{-1} P\left\{\xi\left(T_{1}+\cdots+T_{n}\right) \geqq n^{1 / 2} a_{n}\right\}<\infty .
$$

This is done as follows:

$$
\begin{aligned}
\sum_{n=1}^{\infty} n^{-1} P\left\{\xi\left(T_{1}+\cdots+T_{n}\right) \geqq n^{1 / 2} a_{n}\right\} & \\
= & \sum_{n=1}^{\infty} n^{-1} P\left\{\xi\left(T_{1}+\cdots+T_{n}\right) \geqq n^{1 / 2} a_{n}, \frac{\left|T_{1}+\cdots+T_{n}-n\right|}{n} \leqq \delta\right\} \\
& \quad+\sum_{n=1}^{\infty} n^{-1} P\left\{\xi\left(T_{1}+\cdots+T_{n}\right) \geqq n^{1 / 2} a_{n}, \frac{\left|T_{1}+\cdots+T_{n}-n\right|}{n}>\delta\right\} .
\end{aligned}
$$

First we will show the convergence of the first series in (2.7).

$$
\begin{aligned}
\sum_{n=1}^{\infty} n^{-1} P\left\{\xi\left(T_{1}+\cdots+T_{n}\right)\right. & \left.\geqq n^{1 / 2} a_{n}, \frac{\left|T_{1}+\cdots+T_{n}-n\right|}{n} \leqq \delta\right\} \\
& \leqq \sum_{n=1}^{\infty} n^{-1} P\left(\sup _{n-\delta n \leqq \tau \leqq n+\delta n} \xi(\tau) \geqq n^{1 / 2} a_{n}\right) \\
& \leqq \sum_{n=1}^{\infty} 2 n^{-1} P\left(\xi(1) \geqq \frac{n^{1 / 2} a_{n}}{(n+\delta n)^{1 / 2}}\right)
\end{aligned}
$$

by Lemma (2.5)

$$
\leqq C \sum_{n=1}^{\infty} n^{-1} \exp \left[-\left(\frac{a_{n}^{2}}{2(1+\delta)}\right)(1+o(1))\right]
$$

by the standard Gaussian tail estimate

$$
=C \sum_{n=1}^{\infty} \frac{1}{n}\left(\frac{1}{\lg n}\right)^{(1+\varepsilon)^{2}(1+o(1)) /(1+\delta)}<\infty
$$

if $\delta<\varepsilon$. Now we will show the convergence of the second series in (2.7).

$$
\begin{aligned}
\sum_{n=1}^{\infty} n^{-1} P\left\{\xi\left(T_{1}+\cdots+T_{n}\right)\right. & \left.\geqq n^{1 / 2} a_{n}, \frac{\left|T_{1}+\cdots+T_{n}-n\right|}{n}>\delta\right\} \\
& \leqq \sum_{n=1}^{\infty} n^{-1} P\left\{\left|T_{1}+\cdots+T_{n}-n\right|>\delta n\right\}<\infty
\end{aligned}
$$

by Theorem (2.4) since $E\left(T_{1}\right)=1$. Thus both series in (2.7) converge and the theorem is proved. 


\section{REFERENCES}

1. L. Baum and M. Katz, Convergence rates in the law of large numbers, Trans. Amer. Math. Soc. 120 (1965), 108-123. MR 33 \#6679.

2. L. Breiman, Probability, Addison-Wesley, Reading, Mass., 1968. MR 37 \#4841.

3. J. Davis, Convergence rates for probabilities of moderate deviations, Ann. Math. Statist. 39 (1968), 2016-2028. MR 38 \#3903.

4. W. Feller, An introduction to probability theory and its applications. Vol. II, Wiley, New York, 1966. MR 35 \#1048.

5. I. Gihman and A. Skorohod, Introduction to the theory of random processes, "Nauka", Moscow, 1965; English transl., Saunders, Philadelphia, Pa., 1969. MR 33 \#6689; 40 \#923.

6. P. Hartman and A. Wintner, On the law of the iterated logarithm, Amer. J. Math. 63 (1941), 169-176. MR 2, 228.

7. D. Kostka, On Khintchine's estimate for large deviations, Ann. of Probability 1 (1973), 509-512.

8. J. Lamperti, Probability. A survey of the mathematical theory, Benjamin, New York, 1966. MR 34 \#6812.

9. V. V. Petrov, On a relation between an estimate of the remainder in the central limit theorem and the law of the iterated logarithm, Teor. Verojatnost. i Primenen. 11 (1966), 514-518=Theor. Probability Appl. 11 (1966), 454-458. MR 35 \#3720.

10. F. Spitzer, A combinatorial lemma and its applications to probability theory, Trans. Amer. Math. Soc. 82 (1956), 323-339. MR 18, 156.

11. V. Strassen, $A$ converse to the law of the iterated logarithm, $\mathrm{Z}$. Wahrscheinlichkeitstheorie und Verw. Gebiete 4 (1965/66), 265-268. MR 34 \#850.

Department of Mathematics, Texas A\&M University, College Station, TEXAS 77843 